\title{
Successful endoscopic submucosal dissection of a large cavernous hemangioma in the colon
}

Endoscopic submucosal dissection (ESD) is regarded as a common treatment for complete resection of early gastrointestinal neoplasms [1]. However, few cases have been reported on ESD for resection of cavernous hemangioma in the digestive tract. Here we present a patient with a globular, pedunculated, cavernous hemangioma in the descending colon that was removed successfully en bloc by ESD without any bleeding ( $\vee$ Video 1 ).

A 50-year-old woman was referred to our hospital for melena. Initial colonoscopy examination revealed a submucosal tumor approximately $20 \mathrm{~mm}$ in diameter in the descending colon, mainly characterized by a soft, globular, pedunculated submucosal lesion with a red-purple nodular surface ( $\mathbf{F i g} \mathbf{1} \mathbf{a}, \mathbf{b})$ ). Further endoscopic ultrasonography indicated that the lesion originated from the submucosa. The lesion showed high echogenicity, mixed with a small anechogenic area on the inside, and with a decreased blood flow signal ( $>$ Fig. $1 \mathbf{c}$ ).

For treatment, we first performed endoscopic incision of the colonic mucosa using a Hybrid knife (Erbe, Tübingen, Germany) after submucosal injection ( $\triangleright$ Fig.

$2 \mathbf{a})$. The submucosal dissection was very carefully performed to avoid damage to the body of the cavernous hemangioma and a clear field was maintained. The lesion was then successfully removed from the colon wall by ESD, as described previously ( $\mathbf{F i g .} \mathbf{2}$ b, c, e). We used three clips (two from Anrei Medical, Hangzhou, China and one from Micro-Tech, Nanjing, China) for closure of the mucosal defect ( Fig.2d). Finally, histological examina- tion of the resected specimen confirmed cavernous hemangioma ( $\triangleright$ Fig. $2 \mathbf{f}$ ). The patient was discharged with no further symptoms after 2 days of intravenous antibiotic treatment.

In summary, we believe that this is the first published report of a colonic cavernous hemangioma that has been completely removed by ESD.

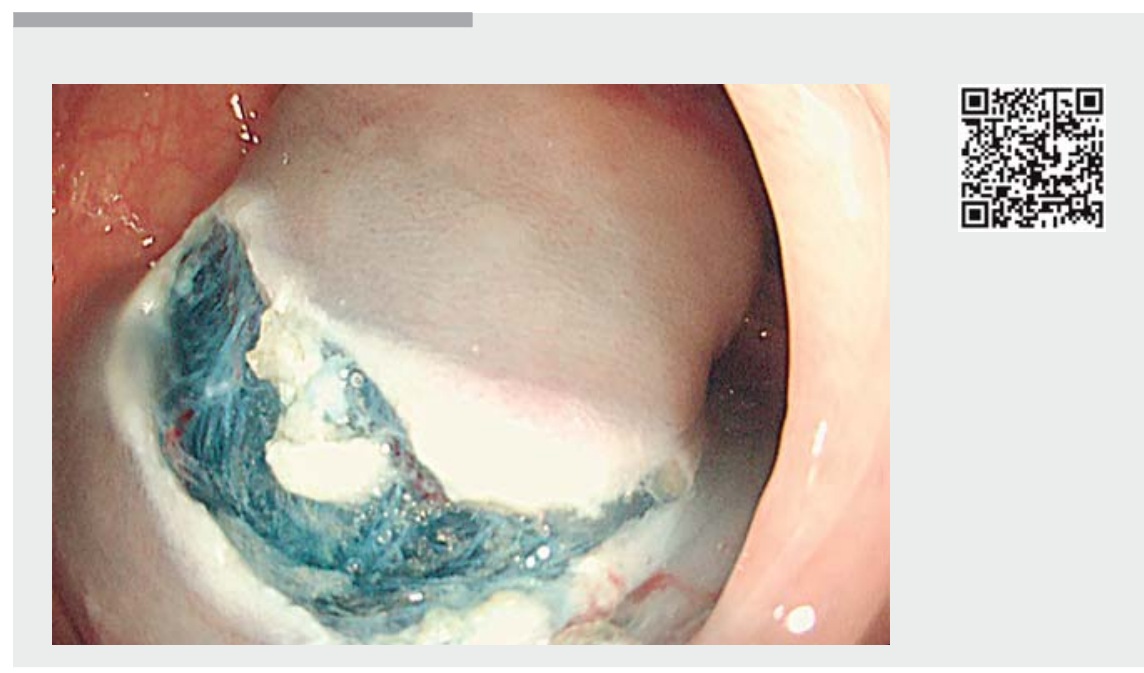

$\checkmark$ Video 1 A large cavernous hemangioma in the colon was successfully removed by endoscopic submucosal dissection.
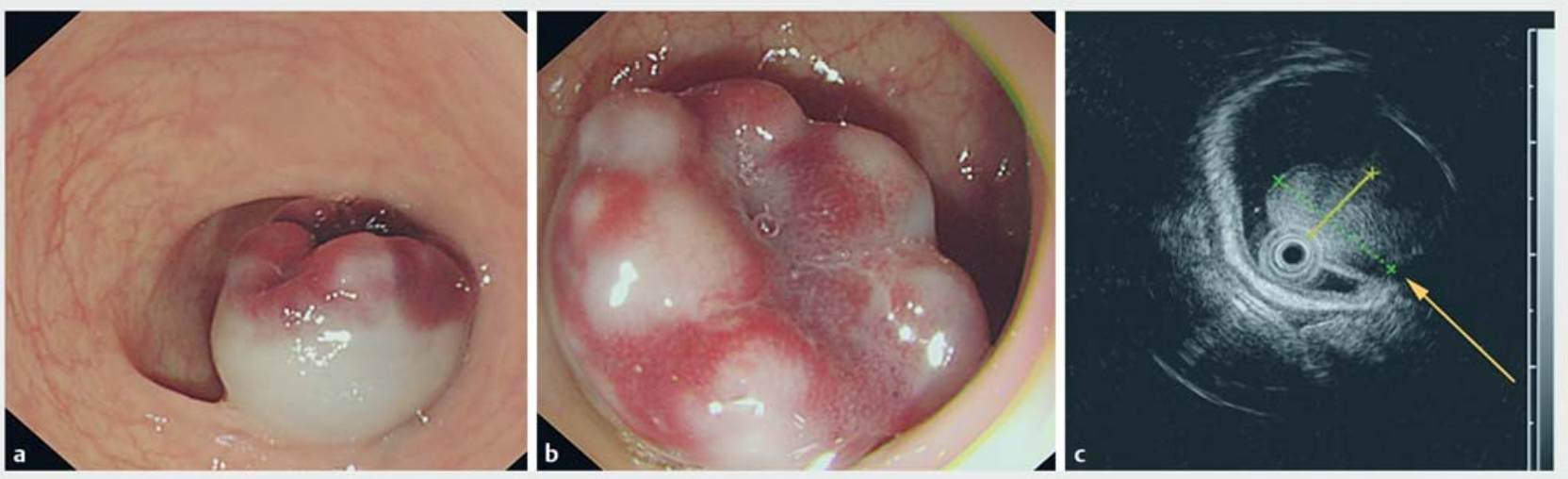

- Fig. 1 Colonoscopy and endoscopic ultrasonography examination of the submucosal lesion. a, b A submucosal tumor approximately 20 mm in diameter characterized by a soft, globular, pedunculated submucosal lesion with a red-purple nodular surface. c Endoscopic ultrasonography indicated that the lesion originated from the submucosa and showed high echogenicity, mixed with a small anechogenic area on the inside, and with decreased blood flow signal (yellow arrow). 

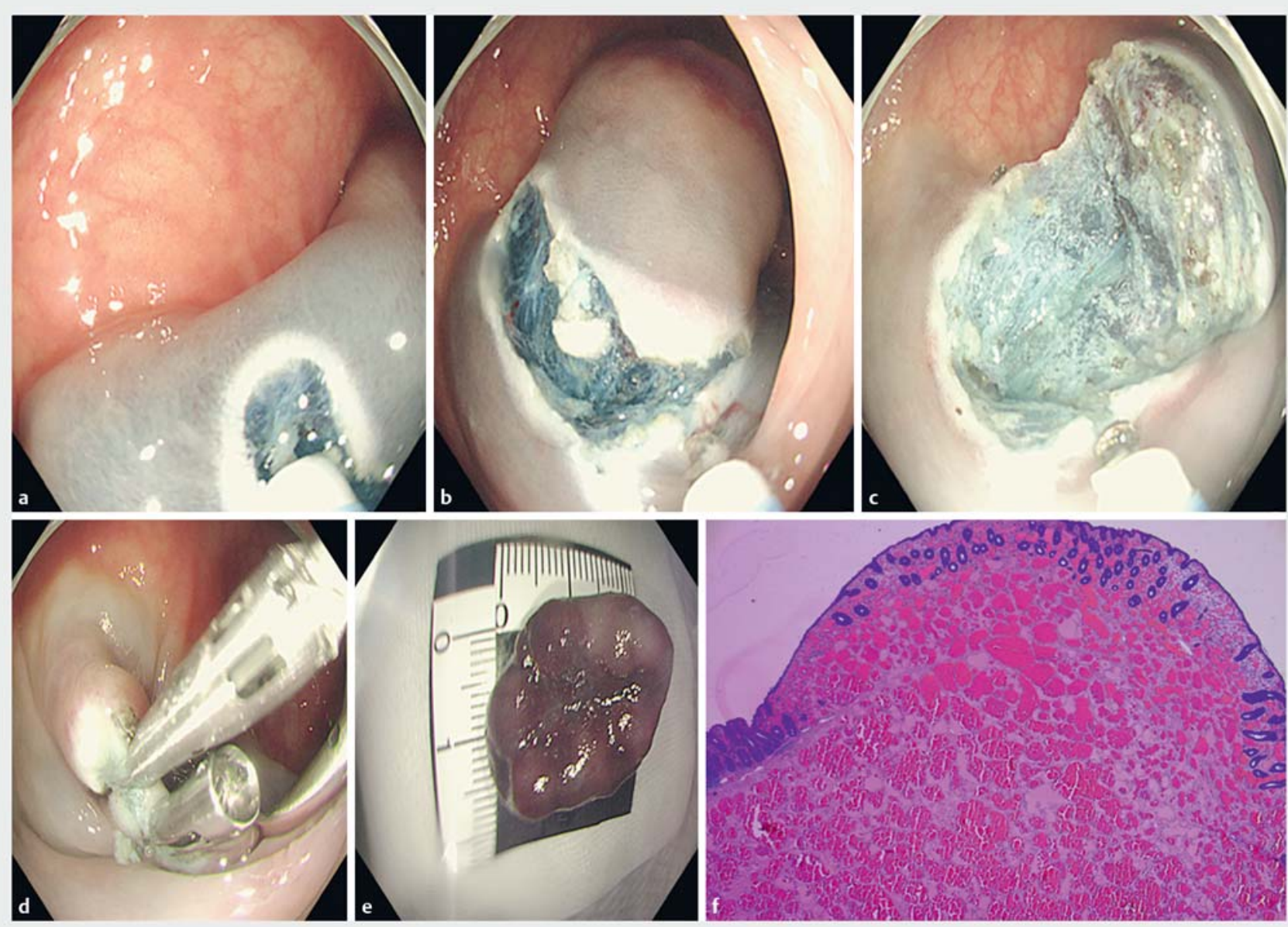

Fig. 2 Colonic cavernous hemangioma was completely removed by endoscopic submucosal dissection (ESD). a An endoscopic incision of the mucosa was performed after submucosal injection. $\mathbf{b}, \mathbf{c}, \mathbf{e}$ The lesion was successfully removed from the colon wall by ESD. $\mathbf{d}$ Closure of the mucosal defect. $\mathbf{f}$ Histological examination of the resected specimen.

Endoscopy_UCTN_Code_CCL_1AD_2AF

\section{Competing interests}

\section{None}

The authors

\section{Kan Chen ${ }^{1, *}$, Min Yan ${ }^{2,}$, Feng Liu ${ }^{1}$}

1 Department of Gastroenterology, Shanghai Tenth People's Hospital, Tongji University School of Medicine, Shanghai, China

2 Department of Pathology, Shanghai Tenth People's Hospital, Tongji University School of Medicine, Shanghai, China

\section{Corresponding author}

\section{Feng Liu, MD}

Department of Gastroenterology, Shanghai Tenth People's Hospital, Tongji University School of Medicine, Middle Yanchang Road No. 301, Jingan District, Shanghai 200072 , China

Fax: +86-21-66300588

drliuffeng@hotmail.com

\section{Reference}

[1] Mavrogenis G, Hochberger J, Deprez P et al. Technological review on endoscopic submucosal dissection: available equipment, recent developments and emerging techniques. Scand J Gastroenterol 2017; 52: $486-498$

\section{Bibliography}

DOI https://doi.org/10.1055/a-0875-3519

Published online: 12.4.2019

Endoscopy 2019; 51: E195-E196

(c) Georg Thieme Verlag KG

Stuttgart · New York

ISSN 0013-726X

\footnotetext{
* These authors contributed equally to this work.
} 